\title{
Acolhimento de gestantes e puérperas suspeitas ou confirmadas de COVID-19 em uma maternidade de referência
}

\author{
Reception of suspected or confirmed pregnant women and mothers of COVID 19 in a \\ reference maternity hospital
}

Recepción de embarazadas y madres sospechosas o confirmadas de COVID-19 en una maternidad de referencia

Josineide de Oliveira Novo França ${ }^{1 *}$, Drielly da Silva Galvão ${ }^{1}$, Aldina lacy Paulain Holanda ${ }^{1}$, Jackeline Vieira Guimarães ${ }^{1}$, Neil Silva de França ${ }^{1}$, Nailu Flor Chenini de Carvalho Reis ${ }^{1}$, Vitória Karoline Maciel Jacauna $^{1}$, Silvani Vieira Cardoso ${ }^{1}$, Maria Suely de Souza Pereira ${ }^{1}$, Alaidistania Aparecida Ferreira ${ }^{1}$.

\section{RESUMO}

Objetivo: Relatar a experiência obtida por residentes de enfermagem obstétrica, frente a nova realidade instalada no atendimento à mulher no ciclo gravídico-puerperal, em uma maternidade, durante a pandemia de COVID-19. Relato de experiência: Foi produzido a partir da vivência das residentes do programa de enfermagem obstétrica vinculado à uma universidade pública do Amazonas que atuaram em uma maternidade de referência no atendimento às mulheres no ciclo gravídico puerperal com suspeita ou confirmação de infecção por COVID-19 no período de junho de 2020 a junho de 2021. A pandemia da COVID19 impôs readequações nos atendimentos das residentes de enfermagem obstétrica às mulheres no ciclo gravídico puerperal assistidas na maternidade, dificuldades enfrentadas pelos profissionais de enfermagem no atendimento às gestantes e puérperas com suspeita ou confirmação de COVID- 19, assim como, o aprimoramento da assistência mediante o novo cenário. Considerações finais: Em decorrência da pandemia de COVID-19 os protocolos de atendimento às mulheres no ciclo gravídico puerperal foram modificados e adaptados, no entendimento de que os profissionais de saúde precisam aplicar estratégias atualizadas para prestar assistência segura e humanizada às pacientes acolhidas na maternidade.

Palavras-chave: Enfermagem obstétrica, Gestantes, COVID-19.

\begin{abstract}
Objective: To report the experience obtained by obstetric nursing residents, facing the new reality installed in the care of women in the pregnancy-puerperal cycle, in a maternity hospital, during the COVID-19 pandemic. Experience report: It was produced from the experience of residents of the obstetric nursing program linked to a public university in Amazonas who worked in a reference maternity hospital in the care of women in the pregnancy-puerperal cycle with suspected or confirmed infection by COVID-19 in the period from June 2020 to June 2021. The COVID-19 pandemic imposed readjustments in the care of obstetric nursing residents to women in the postpartum pregnancy cycle assisted in the maternity hospital, difficulties faced by nursing professionals in the care of pregnant and postpartum women with suspicion or confirmation of COVID-19, as well as the improvement of assistance through the new scenario. Final considerations: As a result of the COVID-19 pandemic, care protocols for women in the pregnancy and puerperal cycle were modified and adapted, on the understanding that health professionals need to apply updated strategies to provide safe and humanized care to patients admitted to the maternity hospital.
\end{abstract}

Keywords: Obstetric nursing, Pregnant women, COVID-19.

1 Universidade Federal do Amazonas (UFAM), Manaus - AM. *E-mail: josyoliveira715@gmail.com

SUBMETIDO EM: 7/2021

ACEITO EM: 7/2021

PUBLICADO EM: 8/2021 


\section{RESUMEN}

Objetivo: Informar la experiencia obtenida por residentes de enfermería obstétrica, frente a la nueva realidad instalada en la atención de la mujer en el ciclo embarazo-puerperal, en una maternidad, durante la pandemia de COVID-19. Informe de experiencia: Se produjo a partir de la experiencia de residentes del programa de enfermería obstétrica vinculadas a una universidad pública en Amazonas que laboraban en una maternidad de referencia en la atención de mujeres en el ciclo de embarazo posparto con sospecha o confirmación de infección por COVID-19 en el período de junio de 2020 a junio de 2021. La pandemia COVID-19 impuso reajustes en la atención de las residentes de enfermería obstétrica a las mujeres en el ciclo de embarazo posparto asistidas en la maternidad, dificultades que enfrentan los profesionales de enfermería en la atención de mujeres embarazadas y posparto con sospecha o confirmación de COVID-19, así como la mejora de la asistencia a través del nuevo escenario. Consideraciones finales: A raíz de la pandemia COVID-19, se modificaron y adecuaron los protocolos de atención a la mujer en el ciclo gestacional y puerperal, en el entendido de que los profesionales de la salud deben aplicar estrategias actualizadas para brindar una atención segura y humanizada a las pacientes ingresadas en el hospital de maternidad.

Palabras clave: Enfermería obstétrica, Mujeres embarazadas, COVID-19.

\section{INTRODUÇÃO}

A COVID-19 é uma doença causada por um novo vírus denominado de Severe Acute Respiratory Syndrome Coronavirus-2 (SARS-CoV-2), oriundo do grupo dos coronavírus, causadores de inflamações respiratórias. Essa doença apresenta um quadro clínico que pode variar entre estado assintomático a grave (MINISTÉRIO DA SAÚDE, 2020a; MINISTÉRIO DA SAÚDE, 2020b).

Os primeiros casos foram registrados na cidade de Wuhan na China, posteriormente em outras cidades do país, em países próximo e logo foi confirmado em outros continentes (PALACIO MAV e TAKENAMI I, 2020). No Brasil, o primeiro caso da COVID-19 foi registrado na cidade de São Paulo, no dia 26 de fevereiro (MINISTÉRIO DA SAÚDE, 2021a). Atualmente, o país já registrou mais de 16 milhões de casos confirmados, mais de 15 milhões de casos recuperados e mais de 460 mil óbitos (MINISTÉRIO DA SAÚDE, 2021c).

Em 11 de março de 2020, a COVID-19 foi caracterizada pela Organização Mundial da Saúde (OMS) como uma pandemia. Os órgãos de saúde divulgaram várias estratégias com orientações para conter o avanço da doença, bem como a epidemia no Brasil, porém essas recomendações têm enfrentado algumas resistências e revelado muitos desafios para os profissionais da saúde, sobretudo em relação às práticas de educação em saúde, realização dos cuidados de enfermagem, Equipamentos de Proteção Individual (EPI), planta física e recursos humanos (PALÁCIO MAV e TAKENAMI I, 2020).

No estado do Amazonas o primeiro caso de COVID-19 foi confirmado no dia 13 de março de 2020, foi um dos estados que mais preocupou as autoridades em saúde, nacionais e mundiais, inicialmente devido a disseminação acelerada do vírus, e consequentemente, a superlotação da rede pública e privada de saúde. Em 23 de março de 2020 o governo do Amazonas decretou estado de emergência, e em abril, os registros oficiais registraram mais de dois mil óbitos. Atualmente, o Amazonas possui mais de 338 mil casos confirmados, mais de 289 mil casos recuperados e mais de 11 mil óbitos (FVS/AM, 2021).

No que tange o atendimento às mulheres em estado gravídico-puerperal, Furlan MCR, et al. (2020), refletem nas constantes mudanças a níveis fisiológicos e imunológicos, percebidas devido às demandas do feto. Logo, é compreensível o estado de vulnerabilidade e susceptibilidade a patógenos, principalmente os que comprometem o sistema respiratório como a SARS-CoV-2, fazendo-se necessários os cuidados apropriados para com as suas especificidades. Carreras SU, et al. (2020) apresentaram que os sintomas manifestados pela gestante são: febre, fadiga, mialgia, tosse seca e dificuldade respiratória, não havendo diferença, portanto, dos sintomas descritos e evidenciados pela população geral.

Um estudo realizado com 427 mulheres grávidas, confirmadas com COVID-19, internadas em hospitais do Reino unido, concluiu que a maioria apresentava sintomas leves, e uma em cada dez necessitou de 
suplementação de oxigênio e internação em ambiente de cuidados intensivos, porém, dado o número de mulheres que necessitaram de cuidados intensivos, provavelmente em lugares em que não se disponha de recursos em quantidade suficiente, os resultados das infecções graves poderiam ser piores (KNIGHT M, et al., 2020).

É importante destacar as alterações de origens psicológicas e emocionais, pois, o fato de gerar uma vida, as novas experiências nas fases gestacional e puerperal, aliado às oscilações hormonais, proporcionam um estado elevado de sensibilidade. Essas modificações emocionais podem aumentar em meio à pandemia, devido ao medo por parte dessas mulheres sobre o real risco de contágio e os efeitos diversos oriundos da infecção (LELIS BDB, et al., 2020; OLIVEIRA MGD, et al., 2019).

A Associação Brasileira de Obstetrizes e Enfermeiros Obstetras (ABENFO), juntamente com suas seccionais fizeram um protocolo das práticas dos enfermeiros obstetras no enfrentamento da pandemia de COVID-19 nas cinco regiões do país através do webinar realizado em cinco de maio de 2020, e trouxe reflexões como: a força da enfermagem obstétrica se destaca no conceito de garantir e potencializar os direitos das mulheres e de provir um cuidado humanizado, respaldado em evidências científicas, destacou a contribuição das enfermeiras residentes para compor as equipes de atuação e para a prestação de um cuidado de qualidade, mesmo em um momento tão atípico como o da pandemia (ALVES VH, et al., 2020).

Diante disso, o presente artigo teve por objetivo relatar a experiência obtida pelas residentes de enfermagem obstétrica, frente à nova realidade instalada no atendimento à mulher no ciclo gravídicopuerperal que procuraram a maternidade durante a pandemia da COVID-19.

\section{RELATO DE EXPERIÊNCIA}

Trata-se de um estudo descritivo, do tipo relato de experiência produzido a partir da vivência das residentes do programa de enfermagem obstétrica vinculado a uma universidade pública do Amazonas que atuaram em uma maternidade de referência no atendimento às mulheres no ciclo gravídico puerperal com suspeita ou confirmação de infecção por COVID-19 no período de junho de 2020 a junho de 2021.

Com o advento da pandemia da COVID-19, houve readequação dos atendimentos na maternidade, atendendo as recomendações da nota técnica no 01/2021 da Agência Nacional de Vigilância Sanitária (ANVISA) (ANVISA, 2021) e da Lei no 13.929/2020 (DOU, 2020). As mulheres suspeitas ou confirmadas de infecção por COVID-19 são provenientes de todo o estado do Amazonas, adentrando a unidade por demanda livre ou reguladas, atendendo as orientações dos órgãos reguladores é instituído o isolamento respiratório e de contato.

No início da pandemia a maternidade dispunha de uma sala rosa e duas suítes cedidas pelo Centro de Parto Normal Intra Hospitalar (CPNI). Com o aumento dos casos em gestantes e puérperas houve a necessidade de perfilizar a maternidade, com entrada para paciente sem sinais e ou sintomas de síndrome gripal distinta da entrada para pacientes com suspeita ou confirmado de COVID-19, assim como para 0 Acolhimento e Classificação de Risco (ACR), admissão, pré-parto, parto e pós-parto, entre outros setores e equipes que ficaram exclusivos para COVID-19.

O fluxo de atendimento compreendia o já estabelecido para assistência das pacientes diferenciado em relação a observação e internação em setores perfilizados, além da realização do teste para deteç̧ão da COVID-19.

As mulheres sintomáticas respiratórias eram identificadas na recepção ou na triagem realizada no ACR pelo(a) enfermeiro(a), sendo encaminhada para o "ACR COVID", acolhimento e classificação de risco específica para COVID-19, a posterior para a sala rosa onde fica aguardando avaliação da equipe, solicitação e coleta de sorologia de anticorpos totais. A identificação precoce dos casos suspeitos ocorria, por meio de metodologia de orientação educativa para os recepcionistas e agentes de portarias, a fim de isolar precocemente a paciente. 
Foram implementadas medidas para mitigar o risco de transmissão, dentre elas podemos citar: suspensão das visitas, uso obrigatório de máscara, manutenção do distanciamento social, orientação sobre a higienização das mãos com água e sabão ou com o álcool em gel da unidade, observando e questionando sobre sintomas gripais ou contactantes sintomáticos.

Foram inúmeras as dificuldades enfrentadas durante a pandemia, pela equipe de saúde, na tentativa de disponibilizar uma assistência mais equânime aos casos suspeitos ou confirmados de COVID-19 como: déficit do serviço regulação do estado, espaço de espera e atendimento não compatível com a demanda, favorecendo a aglomeração, falta de leitos devido à alta demanda. No entanto foi observado um esforço para a aplicação das recomendações na nota técnica n. 04/2020 da ANVISA (ANVISA, 2020) e da Lei $n$. 13.979/2020 (DOU, 2020).

Outra dificuldade observada foi com relação ao atendimento médico, com os casos suspeitos e/ou confirmados de COVID-19, que eram atendidos juntos e sem o critério do atendimento prioritário relacionado às queixas obstétricas. Com o advento de setores exclusivos para atendimento de COVID-19, essa situação veio a ser resolvida seguindo os critérios de prioridade de atendimento instituídos no Manual de Acolhimento e Classificação de Risco em Obstetrícia do Ministério da Saúde de 2017 (MINISTÉRIO DA SAÚDE, 2017).

Buscou-se sempre o propósito de oferecer uma assistência segura e de qualidade, mitigando o risco de contaminação com utilização dos EPI, isolamento das pacientes suspeitas e/ou confirmadas de COVID-19 e perfilização do atendimento.

Após um ano de convivência com a pandemia, muitas condutas foram revistas e alteradas. Na prática, os hospitais passaram a reservar áreas exclusivas para atendimento de COVID-19 desde a sua chegada, isso ocorreu, também, nas maternidades, onde passaram a ter uma área de atendimento para as pacientes infectadas com o SARS-Cov-2 separadas das demais, então, nossa realidade hoje é uma entrada diferenciada para casos suspeitos ou confirmados, desde a criação de recepção, $A C R$, admissão, pré-parto, parto, pós-parto e outros setores exclusivos para essa demanda, fazendo com que tenham um fluxo separado das demais usuárias da maternidade.

Uma ferramenta bastante eficaz foi a educação em saúde continuada e individualizada para cada gestante que chegava à maternidade aos cuidados dos profissionais de enfermagem, que as orientavam, desde a recepção, ao leito e durante os procedimentos, estabelecendo dessa maneira, as relações positivas entre profissional e cliente, gerando segurança e benefícios, tanto para a paciente quanto para os familiares, com aceitação das normas e rotinas da maternidade.

\section{DISCUSSÃO}

A pandemia de COVID-19 tornou-se um problema de saúde pública em todo o mundo sobrecarregando o sistema de saúde e comprometendo a sua capacidade de resposta. Essa tem atingido toda a sociedade, mas é inegável que gestantes e puérperas têm despontado como um grupo de grande preocupação, se no início da pandemia essa população não era considerada de risco, porém com o passar dos meses e o aumento da mortalidade materna devido a casos de COVID-19 passou a ser considerada grupo de risco para desenvolver formas graves da doença (MINISTÉRIO DA SAÚDE, 2020c).

As gestantes assim como idosos e pessoas com comorbidades estão inseridas no grupo de risco, apesar de, ao serem comparadas com a população em geral representarem menor número de contaminação, mas, quando infectadas pelo vírus apresentam desfechos mais graves, e severos diante da vulnerabilidade implicada às mudanças fisiológicas e imunológicas vivenciadas na gravidez. Perante isso, mas também pela imprecisão das reais implicações que o vírus pode causar em gestantes, é preciso um cuidado redobrado, não só no que se refere a contaminação, mas juntamente na condução de uma gravidez o mais saudável possível (SOUZA MEM, et al., 2021).

De acordo com Morais CMS, et al. (2021) no contexto da pandemia o atendimento pré-natal foi afetado, havendo diminuição das consultas presenciais com o intuito de evitar a contaminação por SARS-COV-2. Durante a nossa prática na maternidade deparávamo-nos com muitas mulheres chegavam sem ter consultas 
de pré-natal, outras com poucas consultas, sem os exames sorológicos, ou mesmo sem ultrassonografia obstétrica e todas relataram o mesmo motivo: a dificuldade ou o medo de buscar o serviço de saúde, devido à pandemia. Tais condutas, apesar de compreensíveis vão contra os cuidados recomendados, haja vista que um bom acompanhamento durante o pré-natal, identifica e resolve problemas antes que estes se agravem e tragam riscos a mãe e ao feto (CASTILHOS ED, et al., 2021; SOUZA ASR e AMORIM MMR, 2021).

Para os casos confirmados ou suspeitos a aflição mostrou-se maior, pois, apesar dos avanços, poucos são os conhecimentos científicos das repercussões para o concepto. A infecção por SARS-COV-2 durante a gestação pode acometer tanto a mãe como o feto e a progressão da doença em gestantes estar associada a processo de coagulopatia, com maior possibilidade de desenvolvimento de coágulos intravasculares disseminados nos espaços intervilosos da placenta afetando as trocas gasosas e de nutrientes entre a gravida e o feto. (SOUZA MEM, et al., 2021; MORAIS CMS et al., 2021).

Uma revisão sistemática de 13 artigos sobre COVID-19 e gestação apontou que a infecção por SARSCoV-2 durante a gestação pode causar sofrimento fetal, aborto, parto prematuro e dificuldade respiratória (PANAHI I, et al., 2020). Revisão de literatura realizada por Souza MEM, et al. (2021) apontou que os possíveis impactos da COVID-19 para gestantes e o desenvolvimento foram: parto prematuro, aborto espontâneo, ruptura prematura de membranas, restrição de crescimento intrauterino e sofrimento fetal.

Um estudo no qual fizeram um levantamento dos casos de COVID-19 na população obstétrica desde o primeiro caso documentado no Brasil até o final do estudo, em 18/06/2020, tendo como base o Sistema de Vigilância de Síndromes Respiratórias, se evidenciou 978 gestantes ou puérperas com diagnóstico de Síndrome do Desconforto Respiratório Agudo (SDRA) em decorrência de infecção por COVID-19, destas, 124 evoluíram para óbito, estima-se que esse número seja de 3 a 4 vezes maior do que a mortalidade nessa população por COVID-19 no mundo (TAKEMOTO MLS, 2020).

Quanto aos profissionais de enfermagem, estes seguem as recomendações do Ministério da Saúde - MS, uso dos EPI para atender às pacientes com suspeita ou confirmação de COVID-19, utilizando gorro, máscara, avental, luvas e óculos de proteção, para diminuir o risco de contaminação, foram instituídos setores e profissionais exclusivos para o atendimento das pacientes suspeitas ou confirmadas de COVID-19 (BRASIL, 2021). Vale lembrar que, antes mesmo de haver casos confirmados no Brasil, o sistema de Conselhos Regional e Federal de Enfermagem (Coren/Cofen) lançou uma nota técnica no 01/2020 instruindo os profissionais de enfermagem sobre a utilização correta dos EPI e reiterando a necessidade de as instituições de saúde garantirem a estrutura, equipamentos e materiais adequados para prestação de cuidados (COFEN/COREN, 2020).

A pandemia de COVID-19 proporcionou diversas estratégias e mudanças nas práticas assistenciais. Com essa nova realidade da assistência, surgiu a exigência de criação e/ou adequação de protocolos e condutas nas maternidades. Fez-se necessária uma estratégia de atendimento à gestante mais rápida e eficaz, observando tanto os sintomas da COVID-19, como as queixas obstétricas pela classificação de risco em obstetrícia do Ministério da Saúde. Por tratar-se de algo novo, diariamente fomos desafiados a buscar a melhor forma de prestar um atendimento seguro e de qualidade, buscando respeitar os direitos das mulheres referentes às boas práticas no parto e nascimento, isso, nos proporcionou crescimento profissional, pessoal ao adaptarmos o nosso fazer a realidade imposta pela pandemia.

\section{REFERÊNCIAS}

1. ALVES VH, et al. Enfermagem obstétrica e sua força de trabalho em tempos de covid-19: Relato de experiência das regiões do brasil. Revista Enfermagem em Foco, 2020; 11(2): 103-108.

2. AGÊNCIA NACIONAL DE VIGILÂNCIA SANITÁRIA (ANVISA). Nota técnica GVIMS/GGTES/ANVISA № $01 / 2021$. Brasil, 2021. Disponível em: https://www.gov.br/anvisa/pt-br/centraisdeconteudo/publicacoes/servicosdesaude/notastecnicas/nota-tecnica-01-2021-formularios-iras-2021_final.pdf/view. Acessado em: 21 de março de 2021.

3. AGÊNCIA NACIONAL DE VIGILÂNCIA SANITÁRIA- (ANVISA). Nota técnica GVIMS/GGTES/ANVISA № 04/2020 atualizada em 25/02/2021. Brasil, 2020. Disponível em: https://www.gov.br/anvisa/ptbr/centraisdeconteudo/publicacoes/servicosdesaude/notas-tecnicas/nota-tecnica-gvims_ggtes_anvisa-04_2020-2502-para-o-site.pdf/view. Acessado em: 02 de junho de 2021. 
4. CARRERAS SU, et al. Caracterización clínico-epidemiológica de las gestantes sospechosas y positivas a la COVID19. Revista Cubana de Medicina Militar. 2020; 49(3): e0200800.

5. CASTILHOS ED, et al. Impact of the COVID-19 pandemic on Prenatal, Diabetes and medical appointments in the Brazilian National Health System. 2021: e210013.

6. CONSELHO FEDERAL DE ENFERMAGEM/CONSELHO REGIONAL DE ENFERMAGEM (COFEN/COREN). Nota Técnica № 01/2020: Orientações sobre o novo coronavírus (COVID-19). Revista Enfermagem em Foco, 2020; 11(1): 8-9.

7. DIÁRIO OFICIAL DA UNIÃO (DOU). Lei № 13.979, de 6 de fevereiro de 2020. Disponível em: https://www.in.gov.br/en/web/dou/-/lei-n-13.979-de-6-de-fevereiro-de-2020-242078735. Acessado em: 23 de julho de 2020.

8. FUNDAÇÃO DE VIGILÂNCIA SANITÁRIA (FVS) - Amazonas. Painel Coronavírus. Brasil, 2021. Disponível em: http://www.fvs.am.gov.br/indicadorSalaSituacao_view/60/2. Acessado em: 21 de março de 2021

9. FURLAN MCR, et al. Gravidez e infecção por Coronavírus: desfechos maternos, fetais e neonatais - Revisão sistemática. Revista Cuidarte, 2020; 11(2): e1211.

10. KNIGHT M, et al. Characteristics and outcomes of pregnant women admitted to hospital with confirmed SARS-Cov-2 infection in UK: national population-based cohort study. Revista The BMJ. 2020; 369: m2107.

11. LÉLIS BDB, et al. O Sofrimento Mental das Gestantes em Meio a Pandemia do Novo Coronavírus no Brasil. Revista Multidisciplinar e de Psicologia, 2020; 14(52): 442-451.

12. MINISTÉRIO DA SAÚDE. Doença pelo Novo Coronavírus 2019 - COVID-19. Brasil, 2020a. Disponível em: https://www.saude.gov.br/images/pdf/2020/fevereiro/21/2020-02-21-Boletim-EPIdemiologico03.pdf. Acessado em: 01 de agosto de 2020.

13. MINISTÉRIO DA SAÚDE. O que é COVID-19. Brasil, 2020b. Disponível em: https://www.gov.br/saude/ptbr/coronavirus/o-que-e-o-coronavirus. Acessado em: 01 de agosto de 2020.

14. MINISTÉRIO DA SAÚDE. Painel Geral. Painel Coronavírus. Brasil, 2021c. Disponível em: https://covid.saude.gov.br/. Acessado em: 02 de junho de 2021

15. MINISTÉRIO DA SAÚDE. Secretaria de Atenção à Saúde. Manual de acolhimento e classificação de risco em obstetrícia. $\quad$ Brasil, $2017 . \quad$ Disponível em: https://bvsms.saude.gov.br/bvs/publicacoes/manual_acolhimento_classificacao_risco_obstetricia_2017.pfd. Acessado em: 15 de maio de 2021

16. MINISTÉRIO DA SAÚDE. Secretaria de Atenção Primária à Saúde. Protocolo de manejo clínico do Coronavírus (COVID-19) na atenção primária à saúde. Brasil, 2020c. Disponível em: https://saude.rs.gov.br/upload/arquivos/202004/14140606-4-ms-protocolomanejo-aps-ver07abril.pdf. Acessado em: 23 de julho de 2020.

17. MORAIS CMS, et al. Manifestações dos distúrbios hipertensivos da gravidez e complicações associadas à infecção por COVID-19. Revista Eletrônica Acervo Científico, 26, e7544.

18. OLIVEIRA MG, et al. Sentimento de mulheres com depressão pós-parto frente ao aleitamento materno. Revista Enfermagem em Foco, 2019; 10(3): 88-92.

19. PALÁCIO MAV, TAKENAMI I. Em tempos de pandemia pela COVID-19: o desafio para a educação em saúde. Revista Visa em Debate, 2020; 8(2): 10-15.

20. PANAHI L, et al. Risks of Novel Coronavirus Disease (COVID-19) in pregnancy; a narrative review. Arch Acad Emerg Med, 2020; 8(1): e34

21. SOUZA ASR, AMORIM MMR. Mortalidade materna pela COVID-19 no Brasil. Revista Brasileira de Saúde Materno Infantil, 2021; 21 (Supl. 1): S257-S261.

22. SOUZA MEM, et al. Os possíveis impactos do Sars-CoV-2 em gestantes e no desenvolvimento fetal: revisão narrativa. Revista Eletrônica Acervo Saúde, 2021; 13(6): e8131.

23. TAKEMOTO MLS et al. The tragedy of COVID-19 in Brazil: 124 maternal deaths and counting. International Journal Gynecology \& Obstetrics, 2020; 151(1): 154-156. 